\title{
Counting drugs to understand the disease: The case of measuring the diabetes epidemic
}

\author{
Henrik Støvring*1, Morten Andersen ${ }^{1}$, Henning Beck-Nielsen², \\ Anders Green ${ }^{3}$ and Werner Vach ${ }^{4}$
}

\begin{abstract}
Address: ${ }^{1}$ Research Unit of General Practice, University of Southern Denmark, J.B. Winsløwsvej 9A, 5000 Odense C, Denmark, ${ }^{2}$ Diabetes Research Center, Odense University Hospital, Kløvervænget 64, 5000 Odense C, Denmark, ${ }^{3}$ Department of Epidemiology, University of Southern Denmark, J.B. Winsløwsvej 9B, 5000 Odense C, Denmark and ${ }^{4}$ Department of Statistics, University of Southern Denmark, Campusvej 55, 5230 Odense, Denmark
\end{abstract}

Email: Henrik Støvring* - hstovring@health.sdu.dk; Morten Andersen - mandersen@health.sdu.dk; Henning Beck-Nielsen - henning.becknielsen@ouh.fyns-amt.dk; Anders Green - agreen@health.sdu.dk; Werner Vach - wvach@stat.sdu.dk

* Corresponding author

Published: 2I February 2007

Population Health Metrics 2007, 5:2 doi:10.1 186/1478-7954-5-2
Received: 29 September 2006

Accepted: 2I February 2007

This article is available from: http://www.pophealthmetrics.com/content/5///2

(c) 2007 Støvring et al; licensee BioMed Central Ltd.

This is an Open Access article distributed under the terms of the Creative Commons Attribution License (http://creativecommons.org/licenses/by/2.0), which permits unrestricted use, distribution, and reproduction in any medium, provided the original work is properly cited.

\begin{abstract}
Background: Diabetes prevalence increases globally with severe consequences for afflicted individuals and societies. Data on diabetes incidence and diabetes related mortality on a population level are, however, scarce. As an alternative to dedicated studies it has been suggested to use pharmacoepidemiological databases that are readily available, at least in the Nordic countries.

Methods: For all 470,000 inhabitants in Funen County, Denmark, in the period 1992-2003, data on gender, date of birth, death and migration to and from the county, and any filled prescriptions of an anti-diabetic medication was obtained from the Odense Pharmaco-Epidemiological Database.

Results: Prevalence odds for use of an anti-diabetic medication rose annually $3.5 \%$ (95\% confidence interval: $3.1 \%$, 3.9\%) for females, $4.5 \%$ (4.0\%, 4.9\%) for males. Corresponding incidence rates annually rose $4.8 \%(3.8 \%, 5.8 \%)$ for females, $4.5 \%$ (3.5\%, $5.4 \%)$ for males. Mortality rates among treated annually declined $2.8 \%(1.4 \%, 4.1 \%)$ among females, $2.2 \%(0.9 \%, 3.5 \%)$ among males. The disequilibrium in absolute numbers between incidence and mortality among treated was the main driver for the increasing prevalence, while concurrent trends in incidence and diabetes related mortality only marginally affected prevalence trends. Trend estimates were insensitive to varying the length of the run-in period used for determining treatment status, except when using the naive and methodologically flawed run-in period of variable length.

Conclusion: While pharmacoepidemiological databases provide a useful tool for monitoring pharmacologically treated diabetes, a dedicated diabetes database covering all prevalents and incidents is needed for a more detailed analysis of underlying causes and trends.
\end{abstract}

\section{Background}

The population epidemiology of diabetes mellitus is of public health interest from several perspectives. Diabetes has severe costs for afflicted patients, as it is a chronic lifelong disease characterized by excess mortality [1] and high comorbidity with cardiovascular disease, nephropa- 
thy and eye complications as prominent examples [2]. Diabetes has a high impact on general populations, in particular in Western societies: The prevalence increases with age and reaches a level of about $10 \%$ at the age of 60 years and above in most populations [1]. Diabetes prevalence has been found to increase in all age groups during the last 20 years, and WHO has estimated that the number of diabetics in the world will more than double within the next 25 years from about 170 million in 2000 to about 366 million in year 2030 [3]. This increase of about 3.1\% per year in prevalence has been claimed to fulfill the criteria for an epidemic, particularly of type 2 diabetes $[4,5]$. Others have claimed that an increase in prevalence alone cannot be taken as proof of an ongoing epidemic [6]. Also, prevention of diabetes (in particular type II) has attracted much attention with focus on the relation between lifestyle and risk of becoming a diabetic, and several countries have set up large scale prevention programs. From a medical point of view, the secondary prophylaxis is of high interest with focus on improving life quality and survival with diabetes through health initiatives ranging from motivational intervention, monitoring of disease, to new pharmaceutical treatments.

At the heart of these perspectives is the need to obtain valid and current estimates on trends in diabetes incidence, prevalence and mortality. Such estimates are, however, typically costly to obtain on a general population level, and are hence often missing. In this context, it has been suggested that pharmacoepidemiological databases could be used to study the dynamics of the diabetes epidemiology for large, well-defined populations, even when the databases only contain information on filled prescriptions [7]. A prime advantage of using pharmacoepidemiological databases for such epidemiological descriptions is obviously that the data are readily available. But, although other studies have shown that diabetes prevalence may be reliably estimated from total consumption data $[8-10]$, the use of individual claims data for a more detailed analysis of trends in incidence, prevalence and mortality has so far received less attention. Such an approach is not without its challenges, since both date of onset as well as true, current disease status are not recorded in this type of pharmacoepidemiological databases. Misclassification is thus a potential problem, and the type of potential bias introduced by different strategies for determining treatment status at any given point in time must be carefully considered. Indeed, intuitively attractive approaches may introduce strong biases when studying calendar time trends, as discussed below. Although we update and extend previously published results on the epidemiology of diabetes in the Funen population [7], the primary objective of this paper is methodological. First, we investigate the impact of different choices of run-in periods, as this is a crucial methodolog- ical issue in many pharmacoepidemiological studies. Secondly, we study the relative contributions of current trends in incidence and mortality to the concurrent, observed rise in diabetes prevalence, ie. we focus on the question: Can prevalence rise while contemporary incidence and mortality rates remain constant?

\section{Methods}

Odense Pharmaco-Epidemiologic Database (OPED) was used as data source. OPED contains information on all redemptions of subsidized and prescribed drugs at community pharmacies in the county of Fyn, Denmark [11] since 1992. It is a copy of the exhaustive electronic register maintained for administering all subsidies to pharmacies as well as prescribing physicians in the county. Information on the drug is entered directly into the electronic register when a prescription is processed and dispensed which yields very high validity [12] - and in accordance with the Anatomical Therapeutical Chemical (ATC) classification system [13]. All anti-diabetic drugs are characterized by the first three characters being A10, insulin by A10A, oral anti-diabetics by A10B.

OPED contains information on birth date, gender, migration and death for all subjects living within the county in the period January 1, 1992 to December 31, 2003 in accordance with the Danish Central Person Register. All records allow unique tracking of individuals by means of the nationwide Civil Registration Number.

For each individual we determined presence and treatment status with respect to use of anti-diabetic medications on January 1 of each calendar year (the index date). We defined treatment status based on a previous run-in period's recordings of anti-diabetic drug dispensing, ie. if a subject had at least one prescription within the run-in period, the person was considered treated at the index date. Consequently, for each calendar year we excluded all who immigrated into Fyn during the run-in period, since we could not determine treatment status with certainty for immigrants without an observed redemption.

For those present at the index date and with a sufficient run-in period, we computed annual counts of these entities: prevalence $(\mathrm{P})$, incidence $(\mathrm{I})$, treatment cessation $(C)$, deaths among all non-prevalents $\left(D_{0}\right)$, deaths among prevalents $\left(D_{1}\right)$, and deaths among incidents $\left(D_{\text {Inc }}\right)$. An individual was considered prevalent on the index date if a redemption was observed in the associated run-in period. Incidence was defined as the number of subjects having a redemption in the year of interest, but not in the run-in period. Mortality counts were number of deaths in the year of interest stratified on prevalence status at the index date. Among those prevalent at the index date we defined treatment cessation as having been observed for a period 
which exceeded the length of the run-in period without any new redemptions and which overlapped the index date. In a sub-analysis of the annual transitions between treatment states (treated or not), we further subdivided the number of subjects who had discontinued treatment into two groups: Those who re-initiated treatment within the calendar year, and those who did not.

For incidence and mortality among non-prevalents and prevalents, respectively, we computed rates based on counts and time at risk for the event in question. Time at risk was defined as time from index date to either event or censoring by migration, death, or end of year, whichever was first. Relative mortalities were obtained by comparing mortality rates among prevalents to rates among nonprevalents.

In the sub-analyses of insulin and oral anti-diabetic medications, only redemptions of the specific type were considered; for example, all redemptions of oral anti-diabetic agents were ignored when restricting the analysis to insulin.

Choosing the length of the run-in period is crucial in this study, as in most pharmacoepidemiological studies, since rates of exclusion must be balanced with misclassification. For the fixed run-in periods we did two separate analyses with lengths of one and two years, respectively. The length of one year allows comparison with previously published results [7], but we found it to be inadequate for accommodating a change in the Danish subsidy system taking place in 2000. We thus report on the effects of this change and provide estimates based on a two years run-in period, as these are unaffected by the switch. For illustrative purposes we also present estimates based on increasing the run-in period with calendar time, although these are invalid as they introduce time-dependent misclassification.

As trends in observed incidence of treatment initiation both depends on the true trend in disease incidence as well as trends in detection and tendency to initiate pharmacological treatment, we conducted a sensitivity analysis. Within each year we assume that there is an unknown incidence proportion with an annual trend $\rho$. Further assume that there is a detection probability $\pi_{Y}$, ie. of transition from undiagnosed to becoming diagnosed and initiating pharmacological treatment given that one becomes diabetic. We assumed that $\pi_{1994}$ was $60 \%$. Note that results of the sensitivity analysis with respect to trends are virtually insensitive to the actual level of detection, so this choice is not crucial. For changes in this probability we assumed that they were in the range from a decline of 10 percentage points to an increase of 20 percentage points from 1994 to 2003, ie. that detection proportions in 2003 ranged from $50 \%$ to $80 \%$. This corresponds to annual changes of $-2.0 \%$ to $3.2 \%$ in detection proportions, ie. a ratio $\theta$ of 0.980 to 1.032 between subsequent years. The annual trend $\rho$ in true incidence can now be related to the observed trend $\phi$ in incidence of treatment by the following formula

$\rho=\frac{\phi}{\theta}$

We present graphs showing the relationship between trends in true incidence and the observed trend in treatment incidence when detection proportions vary.

We finally investigated the extent to which the rise in prevalence could be explained by concurrent trends in incidence, mortality among treated and cessation rates, respectively. We did so by comparing the observed annual prevalences to projections based on fixing age-specific rates of incidence, mortality and cessation among treated to their values in 1994. Alternatively, incidence alone was fixed to its level in 1994 to study the isolated impact of a trend in incidence.

\section{Statistical analysis}

Annual incidence, prevalence and mortality rates were determined within four age categories stratified by sex. Annual trend estimates were obtained from regression analysis with year and age categories (cut points at 15, 25, ..., 85) as covariates stratified on gender. Logistic regression was employed for analyzing prevalence, Poisson regression for incidence and mortality rates. In the analyses of prevalence and incidence, correlations among individual's outcomes in subsequent years where allowed for by using robust standard errors $[14,15]$. For prevalence the trend was estimated as a linear trend on the log-oddsratio scale, whereas trends in both incidence and mortality were estimated as linear trends on a log-rate-ratio scale. Cessation rates were not analyzed for trends as they are not of primary interest and in any case estimates would be based on small numbers. Estimates of age-adjusted annual trends with 95\%-confidence intervals are given as odds-ratios and rate-ratios. All analyses were performed in Stata $8[16]$.

\section{Results}

Basic characteristics of the studied population are presented in Tables 1 and 2 using a two year run-in period. Population size is almost constant with only a slight increase while the age-composition changes with an increase in the middle-aged group (40-64 years) and among children $(<15)$, and a decline among younger adults (15-39 years). For both sexes, and in all agegroups, prevalence increases, most distinctly among middle-aged, as does incidence, with males aged 15-39 years 
as the only exception. The total number of deaths among untreated across all age groups declines over the study period, in particular among males above age 65. Mortality among treated increases in absolute numbers, but declines relatively when compared to the concurrent increase in prevalence. A graphical presentation of relative measures is given in Figure 1.

Careful inspection of Table 2 reveals that book-keeping with respect to prevalence does not tally in the following sense: The prevalence of a given year does not correspond to the prevalence plus incidence minus deaths among treated of the previous year. What is missing is the count of apparent treatment cessations. In Table 3 the bookkeeping is carried out including the annual number of treatment cessations and correcting the number of deaths among the prevalents accordingly. The final column of Table 3 shows that the discrepancy between the predicted prevalence according to this book-keeping principle and the actually recorded prevalence is small. The difference is due to censoring induced by migration, since the cohort is open.

Table 4 gives estimates of age- and sex-adjusted trends. Both prevalence and incidence trend estimates are positive and statistically significant, regardless of gender, antidiabetic drug type, and length of run-in. Mortality declines among treated (except for males with respect to insulin when using a two year run-in period) over the study period, and notably also relative to the non-treated population.

\section{Methodological considerations I: Length of run-in}

Table 5 shows the annual count of patients defined as having discontinued treatment based on a gap of either one year or two years between last redemption in the run-in period and first redemption in the following year. Using a gap of one year leads to a peak in 2001 reflecting a change

Table I: Characteristics of source population

\begin{tabular}{|c|c|c|c|c|c|c|c|c|c|c|c|c|c|c|c|c|c|c|c|c|}
\hline \multirow[t]{2}{*}{ Females } & \multicolumn{20}{|c|}{ Age } \\
\hline & \multicolumn{5}{|c|}{$<15$} & \multicolumn{5}{|c|}{$15-39$} & \multicolumn{5}{|c|}{$40-64$} & \multicolumn{5}{|c|}{$65>$} \\
\hline Year & Size & $P$ & 1 & $D_{1}$ & $D_{0}$ & Size & $P$ & 1 & $D_{1}$ & $D_{0}$ & Size & $P$ & I & $D_{1}$ & $D_{0}$ & Size & $P$ & 1 & $D_{1}$ & $D_{0}$ \\
\hline 1994 & $34,5 \mid 4$ & 10 & 0 & 0 & 8 & 73,456 & 339 & 43 & 0 & 39 & 71,305 & 967 & 147 & 12 & 318 & 45,884 & 2,049 & 226 & 197 & 2,081 \\
\hline 1995 & 34,806 & 6 & 3 & 0 & 7 & 72,593 & 343 & 33 & 2 & 34 & 72,133 & 1,046 & 146 & 18 & 313 & 45,862 & 2,069 & 217 & 234 & 2,156 \\
\hline 1996 & 35,398 & 8 & 20 & 0 & 8 & 71,502 & 338 & 46 & 0 & 39 & 73,031 & 1,099 & 163 & 11 & 325 & 45,702 & 2,056 & 271 & 196 & 2,133 \\
\hline 1997 & 35,985 & 23 & II & 0 & 6 & 70,541 & 345 & 27 & 0 & 33 & 73,798 & 1,149 & 154 & 16 & 311 & 45,625 & 2,145 & 226 & 219 & 2,121 \\
\hline 1998 & 36,919 & 32 & 7 & 0 & 6 & 69,832 & 323 & 29 & 2 & 23 & 74,896 & $|, 22|$ & 139 & 16 & 314 & 45,439 & 2,185 & 254 & 211 & 2,043 \\
\hline 1999 & 37,260 & 35 & 9 & 0 & 6 & 69,089 & 303 & 39 & 0 & 22 & 75,568 & 1,234 & 178 & 20 & 323 & 45,472 & 2,264 & 260 & 206 & 2,171 \\
\hline 2000 & 37,627 & 38 & 8 & 0 & 7 & 68,040 & 320 & 41 & I & 28 & 76,436 & 1,293 & 205 & 23 & 310 & 45,407 & 2,346 & 267 & 190 & 2,078 \\
\hline 2001 & 38,124 & 40 & 10 & 0 & 7 & 66,874 & 330 & 49 & I & 24 & 77,218 & $1,4 \mid 2$ & 213 & 27 & 308 & 45,392 & 2,430 & 275 & 187 & 2,057 \\
\hline 2002 & 38,335 & 43 & 12 & 0 & 6 & 66,007 & $34 I$ & 45 & 0 & 36 & 77,832 & 1,500 & 226 & 22 & 276 & 45,571 & 2,562 & 298 & 219 & 2,207 \\
\hline 2003 & 38,420 & 48 & 11 & 0 & 4 & 65,147 & 360 & 78 & 2 & 27 & 78,712 & 1,620 & 253 & 26 & 307 & 45,561 & 2,656 & 314 & 221 & 2,025 \\
\hline \multirow[t]{2}{*}{ Males } & \multicolumn{20}{|c|}{ Age } \\
\hline & \multicolumn{5}{|c|}{$<15$} & \multicolumn{5}{|c|}{$15-39$} & \multicolumn{5}{|c|}{$40-64$} & \multicolumn{5}{|c|}{$65>$} \\
\hline Year & Size & $P$ & 1 & $D_{1}$ & $D_{0}$ & Size & $P$ & 1 & $D_{1}$ & $D_{0}$ & Size & $P$ & 1 & $D_{1}$ & $D_{0}$ & Size & $P$ & I & $D_{1}$ & $D_{0}$ \\
\hline 1994 & 36,066 & 12 & 2 & 0 & 12 & 77,830 & 408 & 49 & I & 83 & 71,598 & 1,424 & 227 & 52 & 478 & 33,454 & 1,658 & 241 & 203 & 1,974 \\
\hline 1995 & 36,486 & 9 & 3 & 0 & 10 & 76,891 & 419 & 45 & 2 & 102 & 72,555 & 1,525 & 212 & 39 & 451 & 33,364 & $1,7 \mid 4$ & 209 & 189 & 2,030 \\
\hline 1996 & 37,173 & 7 & 26 & 0 & 5 & 75,486 & 415 & 55 & 6 & 82 & 73,548 & 1,597 & 250 & 28 & 448 & 33,209 & $1,76 \mid$ & 232 & 197 & 2,057 \\
\hline 1997 & 37,857 & 31 & 5 & 0 & 9 & 74,528 & 423 & 36 & 2 & 87 & 74,466 & 1,739 & 248 & 37 & 455 & 32,994 & $|, 8| \mid$ & 215 & 203 & 1,866 \\
\hline 1998 & 38,839 & 36 & 3 & 0 & 13 & 73,694 & 425 & 40 & 2 & 65 & 75,668 & 1,826 & 292 & 44 & 471 & 32,997 & I,887 & 236 & 192 & 1,857 \\
\hline 1999 & 39,368 & 36 & 8 & 0 & 9 & 72,550 & 430 & 42 & 6 & 86 & 76,434 & 1,956 & 286 & 44 & 455 & 33,087 & 1,974 & 223 & 213 & 1,890 \\
\hline 2000 & 39,741 & $4 I$ & 14 & 0 & 12 & 71,316 & 422 & 41 & 3 & 67 & 77,268 & 2,103 & 315 & 52 & 463 & 33,137 & 2,014 & 253 & 184 & 1,780 \\
\hline 2001 & 39,882 & 46 & 13 & I & 12 & 70,321 & 436 & 51 & I & 47 & 77,965 & 2,227 & 340 & 48 & 444 & 33,425 & 2,158 & 237 & 226 & $\mathrm{I}, 804$ \\
\hline 2002 & 40,034 & 49 & 11 & 0 & 12 & 69,106 & 455 & 64 & 2 & 67 & 78,888 & $2,4 \mid 4$ & 389 & 59 & 477 & 33,655 & 2,213 & 270 & 228 & 1,723 \\
\hline 2003 & 40,093 & 47 & 6 & 0 & 10 & 68,146 & 466 & 43 & 3 & 62 & 79,606 & 2,583 & 401 & 59 & 469 & 34,163 & 2,357 & 333 & 230 & 1,657 \\
\hline
\end{tabular}

Size of population, prevalence, incidence, and mortality with respect to use of anti-diabetic drugs, Fyn County, Denmark, 1994-2003. Size is the size of the population on January I, I993-2003, where subjects migrating into the capture area in the preceding year are ignored. $\mathbf{P}$ is the count of prevalent subjects at January I of the given year. I is the count of incident subjects during the calendar year. $\mathbf{D}_{\mathbf{I}}$ is the count of deaths during the year among subjects prevalent at the beginning of the year. $\mathbf{D}_{0}$ are deaths among non-prevalents in the year. 
Table 2: Characteristics of source population (totals for Table I)

\begin{tabular}{|c|c|c|c|c|c|c|c|c|c|c|}
\hline \multirow[b]{2}{*}{ Year } & \multicolumn{5}{|c|}{ Females } & \multicolumn{5}{|c|}{ Males } \\
\hline & Size & $\mathrm{P}$ & I & $D_{1}$ & $D_{0}$ & Size & $\mathrm{P}$ & I & $D_{1}$ & $D_{0}$ \\
\hline 1994 & 225,159 & 3,365 & 416 & 209 & 2,446 & 218,948 & 3,502 & 519 & 256 & 2,547 \\
\hline 1995 & 225,394 & 3,464 & 399 & 254 & 2,510 & 219,296 & 3,667 & 469 & 230 & 2,593 \\
\hline 1996 & 225,633 & 3,501 & 500 & 207 & 2,505 & 219,416 & 3,780 & 563 & 231 & 2,592 \\
\hline 1997 & 225,949 & 3,662 & 418 & 235 & 2,471 & 219,845 & 4,004 & 504 & 242 & 2,417 \\
\hline 1998 & 227,086 & 3,761 & 429 & 229 & 2,386 & 221,198 & 4,174 & 571 & 238 & 2,406 \\
\hline 1999 & 227,389 & 3,836 & 486 & 226 & 2,522 & 221,439 & 4,396 & 559 & 263 & 2,440 \\
\hline 2000 & 227,510 & 3,997 & 521 & 214 & 2,423 & 221,462 & 4,580 & 623 & 239 & 2,322 \\
\hline 2001 & 227,608 & 4,212 & 547 & 215 & 2,396 & 221,593 & 4,867 & 641 & 276 & 2,307 \\
\hline 2002 & 227,745 & 4,446 & 581 & 241 & 2,525 & 221,683 & $5,|3|$ & 734 & 289 & 2,279 \\
\hline 2003 & 227,840 & 4,684 & 656 & 249 & 2,363 & 222,008 & 5,453 & 783 & 292 & 2,198 \\
\hline
\end{tabular}

Total size of population, prevalence, incidence, and mortality with respect to use of anti-diabetic drugs, Fyn County, Denmark, I994-2003, cf. Table I. Size is the size of the population on January I, 1993-2003, where subjects migrating into the capture area in the preceding year are ignored. $\mathbf{P}$ is the count of prevalent subjects at January $\mathbf{I}$ of the given year. $\mathbf{I}$ is the count of incident subjects during the calendar year. $\mathbf{D}_{\mathbf{I}}$ is the count of deaths during the year among subjects prevalent at the beginning of the year. $\mathbf{D}_{\mathbf{0}}$ are deaths among non-prevalents in the year.

in reimbursement policy in 2000, so apparently some have stockpiled in early 2000 followed by a longer period without redemptions. This effect is smoothed out by using a gap of two years, i.e. the two year run-in period defines treatment status without obvious time-dependent misclassification.

As seen from Table 4 there is, however, virtually no difference in trend estimates of prevalence, incidence and mortality, whether we choose a one or two year year long runin period. Only exception is for incidence, and this is a result of a high incidence in 1993. With a two year run-in period, we are forced to omit this year when estimating trends, and hence the trend is attenuated.

If, however, we were to naively use all available information prior to a given index date to determine treatment status, trend estimates of prevalence become markedly increased, and trend estimates for incidence decreased. The relative biases of this naive approach range from $20 \%-40 \%$ for these parameters. Trends in mortality are unaffected by using a variable length run-in period.

\section{Methodological considerations II: Trends in detection proportions}

In Figure 2 we present the results of our sensitivity analysis. As observed trends in incidence we used the estimated trends of $1.048(1.03 .8,1.058)$ for females, and 1.045 $(1.035,1.054)$ for males. Even for unrealistic large changes in detection proportions the trend in true incidence does not vanish.

\section{Methodological considerations III: Anatomy of an "epidemic"}

Above we observed an increase in prevalence and incidence accompanied by a decrease in mortality among treated. A common interpretation would be to explain the trend in prevalence with the concurrent trends in incidence and mortality. This would however be an oversimplification if prevalence could rise while incidence and mortality rates remained constant. To examine whether this actually occurred during the study period, we compared the observed number of prevalents over the study period to the number of prevalents that would be predicted if incidence, mortality among treated, and cessation rates all had remained constant at their level in 1994. Alternatively, we only fixed incidence rate at its 1994level, while allowing mortality among treated and cessation rates to vary as observed. The resulting projections are shown in Figure 3.

For the period 1995-1999, agreement is very good between observed and projected prevalence among females, while poorer among males. For both genders the discrepancy increases from year 2000. Keeping only incidence fixed improves agreement between observed and projected prevalence substantially, in particular among men, ie. for men the combined decline in mortality and cessation explain more of the observed rise in prevalence than for women. The bulk of the rise in prevalence is thus not due to contemporary changes in incidence or mortality rates, but to incident subjects outnumbering deaths among treated and treatment cessations.

\section{Discussion}

Throughout the study period, prevalence of use of antidiabetic agents increased both in absolute and relative terms, as well as within and across age strata. The corresponding incidence also increased, while mortality among treated declined, also relative to mortality among untreated. This agrees with previous results [7], except with respect to incidence. 

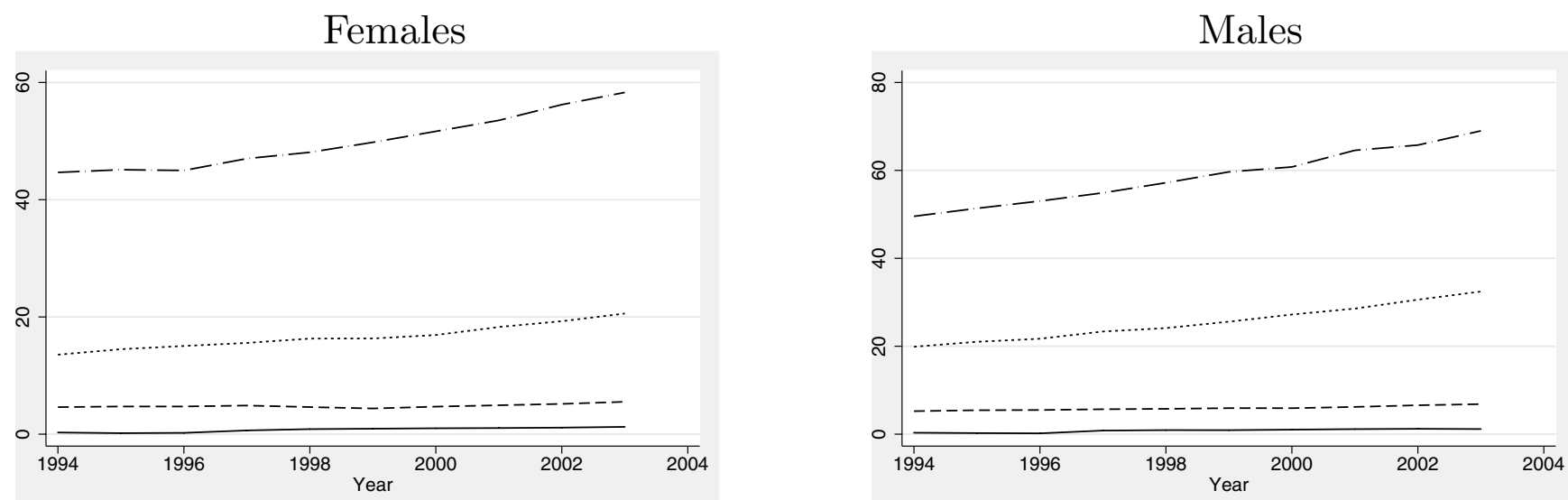

(a) Prevalence per 1,000 persons
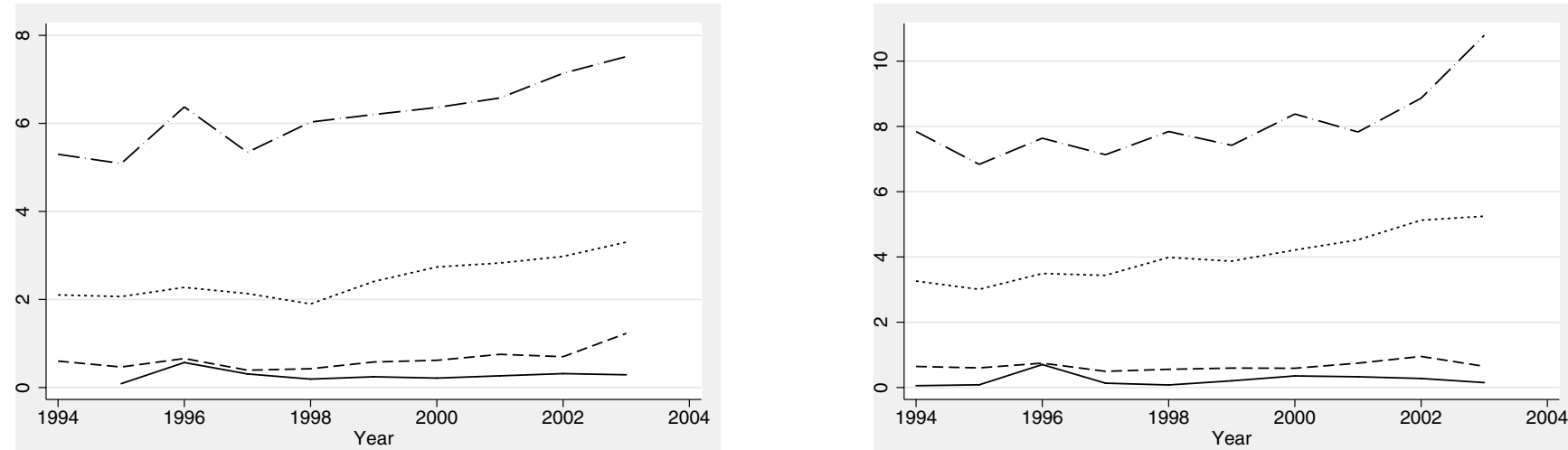

(b) Incidence per 1,000 person-years
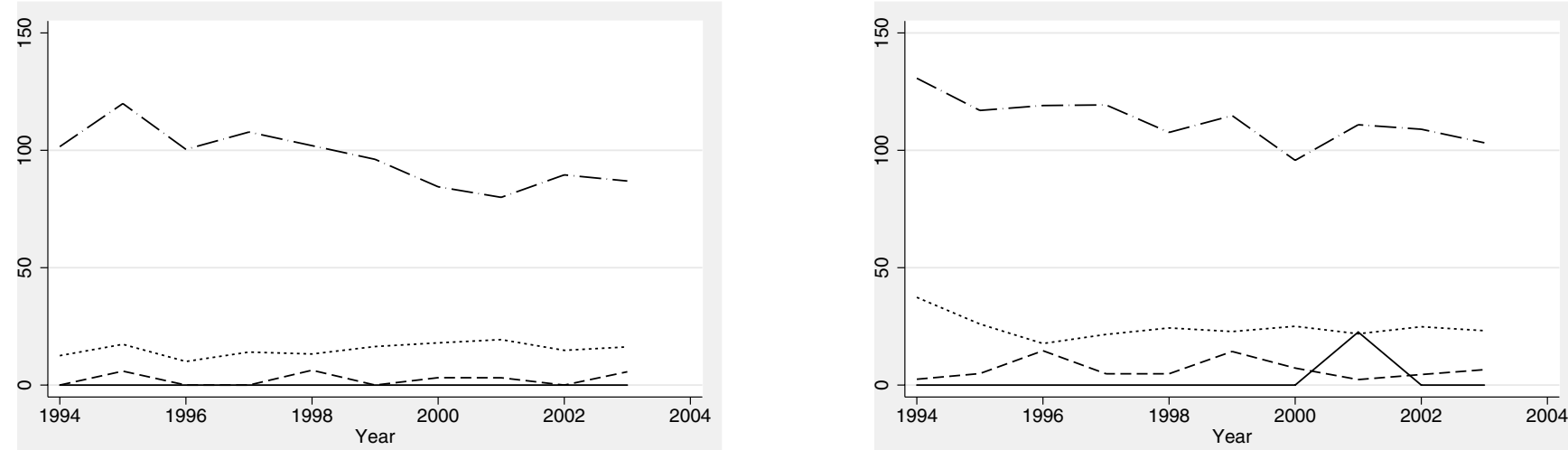

(c) Mortality among treated per 1,000 person-years

\section{Figure I}

Prevalence, incidence and mortality estimates stratified by gender and age, Fyn County, Denmark, 1994-2003. Line-styles indicate age-categories in years: $-0-14,--15-39, \ldots$ 40-64, -- 65+. 
Table 3: Book-keeping for size of total prevalent population

\begin{tabular}{|c|c|c|c|c|c|c|c|c|}
\hline Gender & Year & $P$ & 1 & $C_{N I}$ & $C_{1}$ & $D_{1}$ & $D_{\text {Inc }}$ & Diff \\
\hline \multirow[t]{10}{*}{ Females } & 1994 & 3365 & 416 & 107 & 11 & 207 & 18 & \\
\hline & 1995 & 3464 & 399 & 123 & 10 & 248 & 12 & -3 \\
\hline & 1996 & 3501 & 500 & 109 & 12 & 206 & 22 & 9 \\
\hline & 1997 & 3662 & 418 & 94 & 7 & 230 & 23 & -24 \\
\hline & 1998 & 3761 & 429 & 114 & 9 & 225 & 19 & 5 \\
\hline & 1999 & 3836 & 486 & 91 & 11 & 223 & 14 & -15 \\
\hline & 2000 & 3997 & 521 & 83 & 9 & 209 & 19 & -11 \\
\hline & 2001 & 4212 & 547 & 92 & 8 & 213 & 15 & -14 \\
\hline & 2002 & 4446 & 581 & 96 & 21 & 237 & 20 & -8 \\
\hline & 2003 & 4684 & 656 & 84 & 15 & 244 & 24 & -10 \\
\hline \multirow[t]{10}{*}{ Males } & 1994 & 3502 & 519 & 94 & 5 & 254 & 23 & \\
\hline & 1995 & 3667 & 469 & 109 & 6 & 226 & 18 & -6 \\
\hline & 1996 & 3780 & 563 & 96 & 12 & 228 & 31 & -21 \\
\hline & 1997 & 4004 & 504 & 106 & 7 & 239 & 18 & -15 \\
\hline & 1998 & 4174 & 571 & 105 & 12 & 235 & 26 & 11 \\
\hline & 1999 & 4396 & 559 & 97 & 11 & 261 & 22 & -9 \\
\hline & 2000 & 4580 & 623 & 80 & 17 & 235 & 26 & -17 \\
\hline & 2001 & 4867 & 641 & 96 & 18 & 274 & 21 & -21 \\
\hline & 2002 & 5131 & 734 & 97 & 25 & 288 & 27 & -7 \\
\hline & 2003 & 5453 & 783 & 111 & 15 & 283 & 39 & -27 \\
\hline
\end{tabular}

Bookkeeping for size of total prevalent population with respect to anti-diabetic treatment, Fyn County, Denmark, 1994-2003. P is prevalence on Jan $\mathbf{I}$ of the given year, $\mathbf{I}$ is number of incident subjects, $\mathbf{C}_{\mathbf{N I}}$ is number of persons having discontinued treatment without re-initiation in the given year, $\mathbf{C}_{\mathbf{l}}$ is number of persons having discontinued treatment who re-initiated treatment within the given year, $D_{1}$ is number of deaths among treated, and $D_{\text {Inc }}$ is number of incident subjects dying before the end of the year. Diff for year $Y$ is $P_{Y}-\left(P_{Y-1}+I_{Y-1}-C_{N_{I}, Y_{-1} \mid}-D_{\mid, Y-1}-D_{\text {Inc, } Y_{-1}}\right)$, ie. difference between observed and predicted prevalence. Note, that $\mathbf{C}_{\mathbf{I}}$ does not enter into the computation of Diff since these subjects both leave and re-enter the prevalent pool of subjects in the same year.

The data used are generally of high validity, as they result from automated registration of economic transaction data; near complete coverage, except for medications filled at a pharmacy outside the subjects residential county, which for all drugs was found to not exceed $0.5 \%$ [12]; the information on migration and vital statistics is identical to the relevant subset of the registration at Statistics Denmark.

The major limitation of the present study is its reliance on drug redemptions. Obtaining a drug is not equivalent to using it, and when used there is no observation of end of use. Consequently, we investigated the impact of different treatment classification rules and found that trend estimates for prevalence, incidence, and mortality were largely unaffected, except for the flawed principle of using a run-in period of varying length.

Further, our estimates for prevalence, incidence, and mortality by definition only concern subjects with treated diabetes, i.e. our results do not apply to undiagnosed diabetics, nor to diabetics treated exclusively with diet. These subjects are misclassified in the sense that they are considered part of the general, non-diabetic population in the present study. As all Danish citizens are covered by a government funded health care plan, the annual cost of medications for chronic diseases to be paid by a single individual cannot exceed approximately DKK 3,600 (nearly USD 600) per year. In contrast to many other countries one may therefore expect a rather limited dependence of misclassification rates on social class and, in particular, financial capacity. Other heterogeneities in misclassification rates due to life style, attitudes to health care, etc., may also exist, but like those due to financial capacity and social class, we have little reason to assume that they have changed substantially over the last decade and could imply artificial time trends in incidence or prevalence. Especially, we did not identify the age trend in cessation rates reported by Glynn et al in two studies $[17,18]$. Only for the youngest age-groups were cessation rates slightly elevated, and this may indicate that misclassification may not so much depend on cost as on need and perceived necessity of treatment.

The prevalence level of pharmacologically treated diabetes found here is in line with other contemporary studies in Denmark $[19,20]$. For the year 1996, Kristensen et al [21] found that $71 \%$ of all patients diagnosed with type II diabetes in Vejle County, Denmark, were treated pharmacologically. Further, Drivsholm et al [19] estimated from a one year run-in period the number of pharmacologically treated diabetes patients in Denmark on Dec 31, 1999, to 
Table 4: Trend estimates for incidence, prevalence, and mortality with respect to treatment with anti-diabetic agents

\begin{tabular}{|c|c|c|c|c|c|c|c|c|c|c|}
\hline \multirow{2}{*}{$\begin{array}{l}\text { Run-in } \\
\text { One year }\end{array}$} & \multirow{2}{*}{$\begin{array}{c}\text { Treatment } \\
\text { All anti-diabetic }\end{array}$} & \multirow{2}{*}{$\begin{array}{c}\text { Gender } \\
\text { F }\end{array}$} & \multicolumn{2}{|c|}{ Prevalence } & \multicolumn{2}{|c|}{ Incidence } & \multicolumn{2}{|c|}{ Mortality among treated } & \multicolumn{2}{|c|}{ Relative Mortality } \\
\hline & & & 1.036 & $1.032,1.040$ & 1.045 & $1.035,1.055$ & 0.973 & $0.959,0.987$ & 0.991 & $0.986,0.995$ \\
\hline & & $M$ & 1.044 & $1.040,1.048$ & 1.045 & I.036, I.054 & 0.974 & $0.961,0.987$ & 0.977 & $0.973,0.982$ \\
\hline & Insulin & $\mathrm{F}$ & 1.036 & $1.030,1.043$ & 1.048 & $1.033,1.064$ & 0.977 & $0.955,1.000$ & 0.990 & $0.986,0.994$ \\
\hline & & $M$ & 1.045 & $1.039,1.050$ & 1.067 & I.053, 1.082 & 1.000 & $0.976,1.023$ & 0.977 & $0.973,0.981$ \\
\hline & Oral antidiab. & $\mathrm{F}$ & 1.045 & $1.039,1.051$ & 1.052 & I.042, 1.059 & 0.969 & $0.953,0.986$ & 0.992 & $0.987,0.996$ \\
\hline & & $M$ & 1.052 & $1.046,1.058$ & 1.049 & $1.040,1.058$ & 0.959 & $0.943,0.974$ & 0.979 & $0.975,0.984$ \\
\hline \multirow{6}{*}{ Two year } & All anti-diabetic & $\mathrm{F}$ & 1.035 & $1.031,1.039$ & 1.048 & $1.038,1.058$ & 0.972 & $0.959,0.986$ & 0.990 & $0.986,0.995$ \\
\hline & & $M$ & 1.045 & $1.040,1.049$ & 1.045 & $1.035,1.054$ & 0.978 & $0.965,0.991$ & 0.978 & $0.974,0.982$ \\
\hline & Insulin & $\mathrm{F}$ & 1.034 & $1.028,1.040$ & 1.049 & $1.033,1.065$ & 0.970 & $0.949,0.992$ & 0.990 & $0.986,0.994$ \\
\hline & & $M$ & 1.045 & $1.040,1.051$ & 1.065 & $1.050,1.080$ & 1.005 & $0.983,1.028$ & 0.977 & $0.973,0.982$ \\
\hline & Oral antidiab. & $\mathrm{F}$ & 1.043 & $1.037,1.049$ & 1.055 & $1.045,1.066$ & 0.970 & $0.954,0.987$ & 0.991 & $0.987,0.995$ \\
\hline & & $M$ & 1.052 & $1.046,1.058$ & 1.050 & $1.040,1.060$ & 0.964 & $0.949,0.979$ & 0.980 & $0.976,0.984$ \\
\hline \multirow[t]{2}{*}{ Variable } & All anti-diabetic & $\mathrm{F}$ & 1.046 & $1.042,1.050$ & 1.032 & $1.023,1.04 \mid$ & 0.974 & $0.962,0.985$ & 0.991 & $0.987,0.995$ \\
\hline & & $M$ & 1.053 & $1.049,1.057$ & 1.028 & $1.020,1.036$ & 0.973 & $0.962,0.984$ & 0.979 & $0.975,0.982$ \\
\hline
\end{tabular}

Estimated age-adjusted, annual trends with $95 \%$-confidence intervals for different categories of anti-diabetic drugs and with different lengths of runin periods, Fyn County, Denmark, 1992-2003. For prevalence the trend estimate is given as an odds-ratio based on logistic regression, whereas the remaining estimates are rate-ratios based on Poisson regression. The start of the study period depends on the length of run-in period used.

98,358 , constituting about $75 \%$ of the population of all identified diabetics, estimated to about 130,000 on the same date. Interestingly, Drivsholm et al also found that their primary data sources on hospital discharge diagnosis, measurement of blood glucose, and diabetic foot care, were only able to identify approximately $80 \%-90 \%$ of patients treated pharmacologically for diabetes, indicating the lack of a single data source with complete coverage. In short, it appears reasonable to consider the proportion of pharmacologically treated diabetes patients constant across the study period at about $75 \%$, and to expect the estimated trends in prevalence of pharmacolog-

Table 5: Discontinuation of anti-diabetic treatment

\begin{tabular}{|c|c|c|c|c|c|c|c|c|}
\hline \multirow[b]{3}{*}{ Year } & \multicolumn{4}{|c|}{ Females } & \multicolumn{4}{|c|}{ Males } \\
\hline & \multicolumn{2}{|c|}{ One year gap } & \multicolumn{2}{|c|}{ Two years gap } & \multicolumn{2}{|c|}{ One year gap } & \multicolumn{2}{|c|}{ Two years gap } \\
\hline & $n$ & $\%$ & $n$ & $\%$ & $n$ & $\%$ & $n$ & $\%$ \\
\hline 1993 & 198 & 6.6 & & & 174 & 5.6 & & \\
\hline 1994 & 209 & 6.7 & 118 & 3.5 & 185 & 5.7 & 99 & 2.8 \\
\hline 1995 & 199 & 6.2 & 133 & 3.8 & 188 & 5.5 & 115 & 3.1 \\
\hline 1996 & 172 & 5.3 & $12 \mid$ & 3.5 & 203 & 5.8 & 108 & 2.9 \\
\hline 1997 & 188 & 5.5 & 101 & 2.8 & 196 & 5.2 & 113 & 2.8 \\
\hline 1998 & 163 & 4.6 & 123 & 3.3 & 182 & 4.6 & 117 & 2.8 \\
\hline 1999 & 135 & 3.7 & 102 & 2.7 & 166 & 4.0 & 108 & 2.5 \\
\hline 2000 & 150 & 4.0 & 92 & 2.3 & 194 & 4.5 & 97 & 2.1 \\
\hline 2001 & 282 & 7.1 & 100 & 2.4 & 375 & 8.2 & 114 & 2.3 \\
\hline 2002 & 176 & 4.2 & 117 & 2.6 & 233 & 4.8 & 122 & 2.4 \\
\hline 2003 & 189 & 4.2 & 99 & 2.1 & 250 & 4.9 & 126 & 2.3 \\
\hline
\end{tabular}

Number of patients discontinuing anti-diabetic treatment, Fyn County, Denmark, 1994-2003. Discontinuation is defined as subjects with more than one or two years, respectively, without redemptions after a previous redemption. Percentage figures indicate relative proportion of the prevalent population who discontinues treatment. ically treated diabetes to be valid for the entire diabetes population. Regardless, we suggest our results to be of genuine public health interest in themselves, as they pertain to that group of diabetics which must generally be considered most severely afflicted, and thus requiring most care.

A third consequence of relying on a pharmacoepidemiological database is the inability to control for date of diagnosis in the analysis. In particular, in the analysis of mortality among treated we could not account for duration of disease, and part of the explanation for the apparently decreasing mortality could be earlier treatment of patients with less severe diabetes.

The trend in incidence identified in this study was comparable to the one found for the period 1993-1999, reported in [7], although slightly attenuated. The attenuation occurred both because the year 1993 was excluded in the present study using a two year run-in period, and because incidence began a steeper increase in 2000. Combined with the enlarged sample size due to a longer study period, this made the trend statistically significant. The increase in incidence observed after year 2000 could hallmark the onset of a true diabetes epidemic, possibly reflecting an impact from a sustained obesity epidemic on onset of diabetes or other changes in lifestyle - although postulating a general obesity epidemic in Denmark would also appear to be an over-simplification according to a recent study showing the existence of large heterogeneity in obesity trends [22]. That an epidemic of diabetes could be looming was supported by the finding that fixing incidence at its level in 1994 could predict prevalence with 


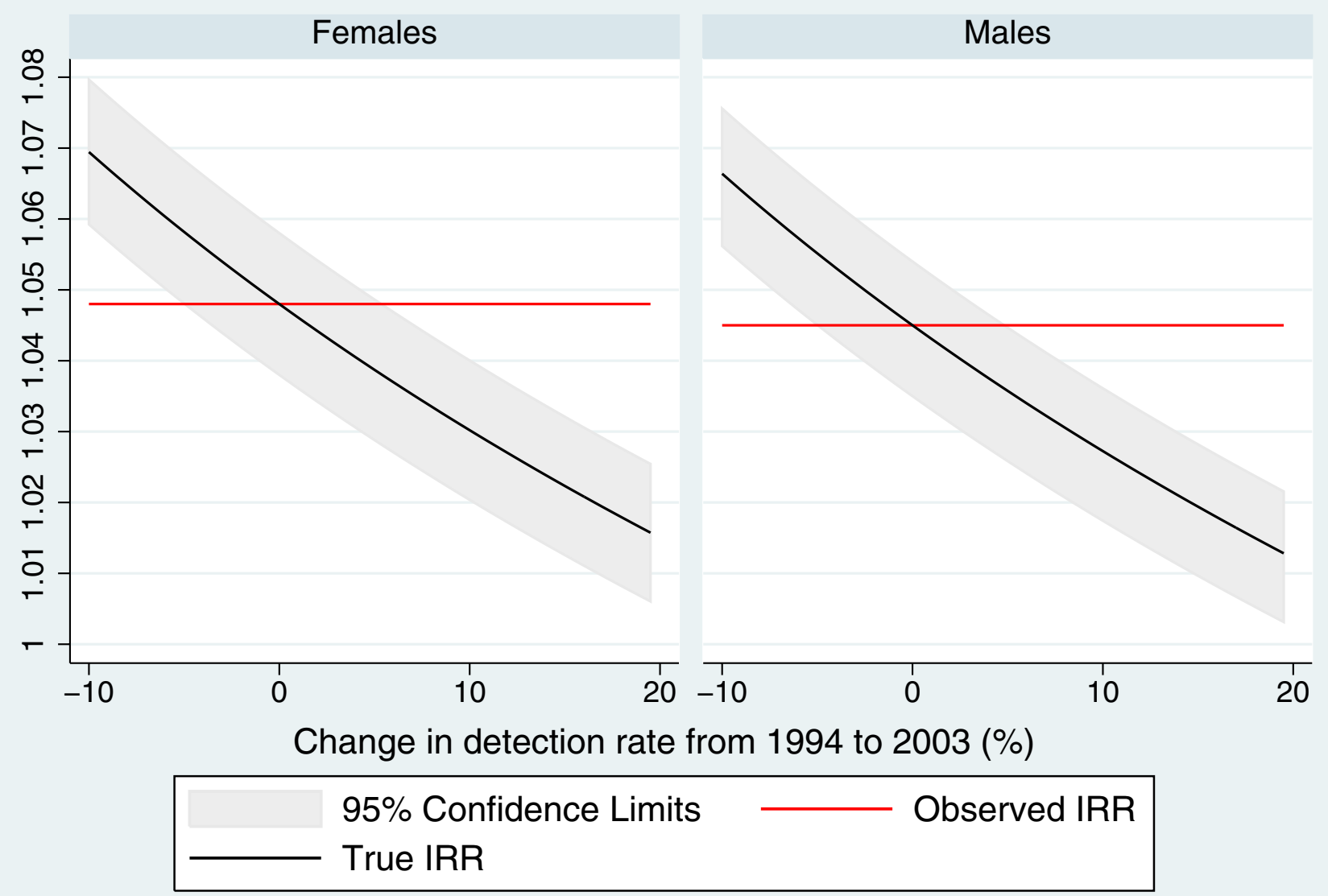

Graphs by Gender

\section{Figure 2}

Observed and true trends in incidence with respect to hypothesized changes in detection proportions from I994 to 2003. Confidence limits for the true trends are computed from the confidence limits of the observed trend by application of Formula I.
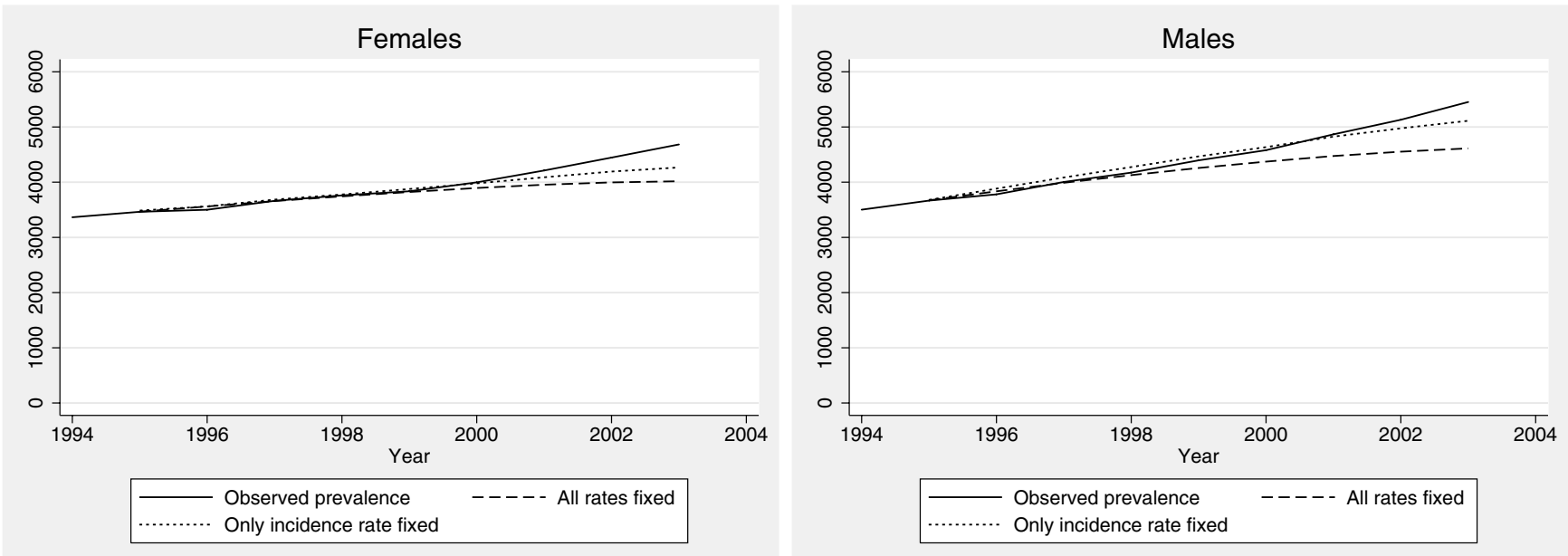

Figure 3

Observed and predicted prevalences with respect to use of anti-diabetic drugs, Fyn County, Denmark, 1994-2003. Fixation of rates is with respect to their 1994-level. 
good precision until 2000, at which point the observed prevalence started to outrun projected prevalence. It may, however, be speculated that new diagnostic criteria, which eased detection of new cases and were implemented in 1999 in Denmark, could have contributed to the observed increase in incidence. For trends in incidence, our somewhat simplistic sensitivity analysis did however show that even unrealistically large changes in detection proportions could not remove the observed trend in incidence entirely. One must however interpret these results cautiously for two reasons. First the detection proportions are by definition a simplified representation of the problem, as they ignore the duration between true onset of disease and initiation of pharmacological treatment. We are however not aware of any studies on this duration, let alone trends in durations, which could be used to further qualify our analysis, as existing studies only report prevalence of undiagnosed diabetes [23]. Secondly, the detection proportion covers both diagnosis and treatment initiation. It might well be speculated that a trend towards earlier diagnosis could be offset by a smaller need for pharmacological treatment among those with earlier diagnosis. For this reason we believe that the studied range of changes in detection proportions is probably too wide to only contain realistic values. Note, that our considerations about the relation of observed incidence, prevalence and mortality are independent of any assumptions about the causal reason for a shift in incidence, be it a real increase in subjects developing diabetes, a change in diagnostic procedures, criteria or habits, or a change in treatment practices.

Whatever the explanation, the trend in incidence should not be considered the primary reason for the concurrent increase in prevalence. Instead, the primary explanation is the annual surplus of subjects initiating treatment for diabetes compared to those leaving treatment which must be considered a legacy of past diabetes incidence and treatment. The surplus may be expected to increase further, if current trends in incidence and mortality persist, and projections by WHO and others may thus severely underestimate the true magnitude of diabetes prevalence ten to twenty years from now, built as they are on stationary agespecific incidence and mortality estimates [3].

In conclusion, we suggest that pharmacoepidemiologic databases can provide useful information on current trends in diabetes epidemiology if analyzed properly, and at a much lower cost than ordinary studies. The major drawback is the lack of insight in the underlying reasons for any observed trends - an insight which can only be obtained on a regular basis if a population-wide case database collecting information on all incident and prevalent diabetes cases is established.

\section{Competing interests}

The author(s) declare that they have no competing interests.

\section{Authors' contributions}

Støvring had the original idea for the study, carried out all analyses and drafted the original and revised manuscripts. Andersen provided access to the data. The planning of analyses and interpretation of the data was the product of discussions involving all authors, all of whom also commented on drafts of the paper. All authors have seen and approved the final version.

\section{References}

I. Harris M, Hadden W, Knowler W, Bennet P: Prevalence of diabetes and impaired glucose tolerance and plasma glucose levels in U.S. population aged 20-74 yr. Diabetes 1987, 36:523-34.

2. Stratton I, Adler A, Neil H, Matthews D, Manley S, Cull C, Hadden D, Turner R, Holman R: Association of glycaemia with macrovascular and microvascular complications of type 2 diabetes (UKPDS 35): prospective observational study. British Medical Journal 2000, 32 I(7258):405-I2.

3. Wild S, Roglic G, Green A, Sicree R, King H: Global prevalence of diabetes: estimates for the year 2000 and projections for 2030. Diabetes Care 2004, 27(5): 1047-53.

4. Mokdad A, Bowman B, Ford E, Vinicor F, Marks J, Koplan J: The continuing epidemics of obesity and diabetes in the United States. JAMA 200I, 286: I 195-200.

5. Colagiuri S, Borch-Johnsen K, Glümer C, Vistisen D: There really is an epidemic of type 2 diabetes. Diabetologia 2005.

6. Green A, Støvring H, Andersen M, Beck-Nielsen H: The epidemic of type 2 diabetes is a statistical artefact. Diabetologia 2005.

7. Støvring H, Andersen M, Beck-Nielsen H, Green A, Vach W: Rising prevalence of diabetes: evidence from a Danish pharmacoepidemiological database. Lancet 2003, 362(9383):537-8.

8. Sartor F, Walckiers D: Estimate of disease prevalence using drug consumption data. Am J Epidemiol I995, I4 I(8):782-787.

9. Papoz L: Utilization of drug sales data for the epidemiology of chronic diseases: the example of diabetes. The EURODIAB Subarea C Study Group. Epidemiology 1993, 4(5):42 I-427.

10. Duarte-Ramos F, Cabrita J: Using a pharmaco-epidemiological approach to estimate diabetes type 2 prevalence in Portugal. Pharmacoepidemiol Drug Saf 2006, I 5(4):269-274.

II. Hallas J, Nissen A: Individualized drug utilization statistics. Analysing a population's drug use from the perspective of individual users. Eur J Clin Pharmacol I994, 47(4):367-372.

12. Gaist $D$, Sorensen $H$, Hallas J: The Danish prescription registries. Dan Med Bull 1997, 44(4):445-8.

13. The WHO Collaborating Centre for Drug Statistics Methodology: ATC index with DDDs and Guidelines for ATC classification and DDD assignment. Oslo 2001.

14. Huber PJ: The behavior of maximum likelihood estimates under nonstandard conditions. In Proc. Fifth Berkeley Sympos. Math. Statist. and Probability (Berkeley, Calif., 1965/66), Statistics Volume I. Berkeley, Calif.: Univ. California Press; 1967:221-233.

15. White $\mathrm{H}$ : Maximum likelihood estimation of misspecifled models. Econometrica 1982, 50:1-25.

16. StataCorp: Stata Statistical Software: Release 8.0. College Station, Texas: Stata Corporation 2003.

17. Glynn RJ, Monane M, Gurwitz JH, Choodnovskiy I, Avorn J: Aging, comorbidity, and reduced rates of drug treatment for diabetes mellitus. J Clin Epidemiol 1999, 52(8):781-790.

I8. Glynn RJ, Monane M, Gurwitz JH, Choodnovskiy I, Avorn J: Agreement between drug treatment data and a discharge diagnosis of diabetes mellitus in the elderly. Am J Epidemiol 1999, I49(6):54|-549.

19. Drivsholm TB, Frederiksen K, de Fine Olivarius N, Ødegaard B, Kristensen JK: [The prevalence of diabetes in Denmark. Development of a method for a registry-based assessment]. Ugeskr Laeger 2003, I65(29):2887-289।. 
20. Danish Medicines Agency: Antidiabetika: Forbruget af antidiabetika 1994 - 1997 (Antidiabetics: The use of antidiabetics 1994 - 1997). Brønshøj, Denmark; 1998. [(In Danish)]

2I. Kristensen JK, Bro F, Sandbaek A, Dahler-Eriksen K, Lassen JF, Lauritzen T: HbAlc in an unselected population of 4438 people with type 2 diabetes in a Danish county. Scand J Prim Health Care 200I, 19(4):24I-246.

22. Heitmann B, Stroger U, Mikkelsen K, Holst C, Sorensen T: Large heterogeneity of the obesity epidemic in Danish adults. Public Health Nutr 2004, 7(3):453-60.

23. Cowie CC, Rust KF, Byrd-Holt DD, Eberhardt MS, Flegal KM, Engelgau MM, Saydah SH, Williams DE, Geiss LS, Gregg EW: Prevalence of diabetes and impaired fasting glucose in adults in the U.S. population: National Health And Nutrition Examination Survey 1999-2002. Diabetes Care 2006, 29(6): | 263-I 268.

Publish with Bio Med Central and every scientist can read your work free of charge

"BioMed Central will be the most significant development for disseminating the results of biomedical research in our lifetime. "

Sir Paul Nurse, Cancer Research UK

Your research papers will be:

- available free of charge to the entire biomedical community

- peer reviewed and published immediately upon acceptance

- cited in PubMed and archived on PubMed Central

- yours - you keep the copyright

Submit your manuscript here:

http://www.biomedcentral.com/info/publishing_adv.asp
BioMedcentral 